\title{
Reliability Calculation of Product Failure Data (Part II : Experiment)
}

\author{
Xintao Xia ${ }^{1, a}$, Yunfei $\mathrm{Li}^{1, b^{*}}$, Zhen Chang ${ }^{1, c}$, Bin Liu ${ }^{1, d}$ and Liang Ye $e^{1, e}$ \\ ${ }^{1}$ Mechatronical Engineering College, Henan University of Science and Technology, Luoyang \\ 471003, China \\ axiaxt1957@163.com, b631617926@qq.com, czhenc1992@163.com, dlbin1992@foxmail.com, \\ e172682823@qq.com \\ ${ }^{*}$ The corresponding author
}

Keywords: Rolling bearing; Failure data; K-s hypothesis testing; Standard deviation; Reliability

\begin{abstract}
On the reliability of failure data, this paper provides wide ranges of theoretical method, and determines the effectiveness of those methods about rolling bearing. Under the reference standard based on the empirical value of medium rank, the veracity about those methods apply to rolling bearing would be determined with the k-s hypothesis testing, and there have a contrastive analysis between the reliabilities and empirical values about different methods, then estimates those methods. For the result of this paper, the veracity of lognormal distribution (L) and Weibull distribution $(\mathrm{W})$ has a great unsteadiness. However, the maximum entropy probability distribution (M) could reply any confirmable distributions, and be suitable for poor information as unknown as the probability distribution, apriori information and trend term. Therefore, the maximum entropy probability distribution has a better applicability for the problem of rolling bearing's reliability on failure data.
\end{abstract}

\section{Introduction}

With the rapid development of science and technology, the requirements of the times have increasing constantly, the standard become stricter. Human have a higher standard for high quality precision equipment. The military project is strictest than others because of its special character in numerous industries. The military power refers to the national security, so the performance requirement on equipment for military is so significance. Rolling bearing is one of the most important components in mechanical equipment, and that's performance would have a great influence about the accuracy of equipment. So it's not allowed to have any issues on the crucial moment, or there have an immensurable loss for person, society and nationality. Due to its particularity, the cost is an enormous number, and the experiment strictly, we could have enough data to analysis the reliability of new product, that causes multitudinous troubles.

At present, there have numerous studies on the method of processing failure data. Schneidewind, Norman F make a model that includes the concept of seiecting a subset of the failure data is the Schneidewind nonhomogeneous Poisson process(NHPP) software reliability model to get the reliability[1]. Marcorin suggest that in order to estimate a product's reliability, parametric inferential methods are required to evaluate survival test data, which happens to be a fairly expensive data source [2]. Zhang study the models involving three Weibull distributions or mixture of them [3]. John describes the application of the Solver function contained within Microsoft Excel to generate solutions to a range of common failure data analysis problems [4-5]. Yang addresses this type of challenging probability density evolution method (PDEM), and presents a Bayesian approach to aggregate expert estimates on human error probabilities to determine the relationships of an HRA model [6]. Hao builds a model uses the penalty function method to deal with the constraint function which is changed from the equality constraint to the inequality one [7]. Li and David study the reliability based on the fuzzy theory [8-9]. Xia provided the grey bootstrap method can be employed to generare many simulated data with few data outputted [10].

In this paper, the analysis of failure data would processing based on the lognormal distribution, Weibull distribution and the maximum entropy probability distribution on reliability of rolling 
bearing. As the benchmark in the empirical value of Johnson method on reliability, there have a comparison between the lognormal distribution of two-parameter and the Weibull distribution of two-parameter, and parameter estimation with maximum likelihood method; then contrast the lognormal distribution with the Weibull distribution of three-parameter, and parameter estimation with probability weighted moment method. The analysis indicates that, lognormal distribution and Weibull distribution aren't suitable for every situations of failure about rolling bearing, and the result is unstable; yet the degree of fitting of maximum entropy probability distribution is perfect, the applicability includes each situation, in addition, the relative error, with different theoretical methods, is lower.

\section{Experimental Investigation and Data Analysis}

Experimental Installation and Condition. The testing machine's size-suit is $\Phi 12 \mathrm{~mm}$, and used point-contact type. In this experiment process, the speed of motor is $3900 \mathrm{r} / \mathrm{min}$, the operating temperature is $26^{\circ} \mathrm{C}$, and the humidity is fifty-three percent. Under loading with compression spring, the value between cylindrical roller and steel ball is $2.55 \mathrm{KN}$, and the contact stress adds to 5.88 GPa.

Experimental Data. The failure data of this test would be distinguished to three groups, one hour as the unit of failure data. The permutation of data each group increasingly set as one vector quantity, " $n$ " presents the number of failure data.

$\mathrm{T} 1=[0.38,1.685,1.687,1.71,1.8,1.86,2.06,2.14,2.2,2.42,2.46,3.88,4.89,6.2,7.73,12.46,12.5,12.88$, $13.33,31.97,38,57,47.5,50.2,51.77,58.71] ; n=26$.

$\mathrm{T} 2=[0.61,0.69,1.66,1.81,1.91,1.93,2.34,2.36,2.38,3.07,3.075,3.08,3.63,11.80,12.67,14.18,14.29,1$ $6.27,17.84,18.83,26.10,28.00,29.79,47.52,47.86,52.91,53.15,53.57,80.20,90.11] ; n=30$.

$\mathrm{T} 3=[1.46,1.685,1.687,1.88,2.06,2.13,2.25,2.257,2.39,2.48,2.58,4.32,4.97,8.55,11.34,12.78,15.75$, 22.66,32.49,69.72,71.54,86.36,86.91]; $n=23$.

Parameter estimation for T1, T2, and T3 based on different theoretical methods, the result would be showed in Table 1.

Table 1 The result of parameter estimation

\begin{tabular}{lccc}
\hline & T1 & T2 & T3 \\
\hline \multirow{2}{*}{ L-Two } & $\mu=1.7859 ;$ & $\mu=2.2075 ;$ & $\mu=1.9509 ;$ \\
& $\sigma=1.3713$ & $\sigma=1.4681$ & $\sigma=1.4091$ \\
\cline { 2 - 4 } W-Two & $\eta=12.0224 ;$ & $\eta=18.6897 ;$ & $\eta=14.7678 ;$ \\
& $\beta=0.7589$ & $\beta=0.7861$ & $\beta=0.6938$ \\
\cline { 2 - 4 } L-Three & $\tau=3.3308 ;$ & $\tau=1.1823 ;$ & $\tau=5.8718 ;$ \\
& $\mu=1.5137 ;$ & $\mu=2.4503 ;$ & $\mu=1.4258 ;$ \\
& $\sigma=1.3358$ & $\sigma=1.0573$ & $\sigma=1.5440$ \\
\hline \multirow{2}{*}{ W-Three } & $\eta=10.5119 ;$ & $\eta=20.4337 ;$ & $\eta=11.7192 ;$ \\
& $\beta=0.6631 ;$ & $\beta=0.8334 ;$ & $\beta=0.5706 ;$ \\
& $\tau=0.3655$ & $\tau=-1.0515$ & $\tau=0.6897$ \\
\hline
\end{tabular}

Through the parameter estimation based on lognormal distribution and Weibull distribution and maximum entropy probability distribution, the result would be shown in Fig. 1 3. According to these figures, the curve of three methods on reliability is nearly accord with empirical distribution, and the degree of fitting exerted is very ideal. The significance level is $\alpha=0.05$ based on the k-s hypothesis testing. The result of hypothesis testing would be shown in Table 2 4 for parameter estimation. This research shows that each k-s test value of three groups of failure data have no over the critical value, and these curves could reflect the regularities of distribution of failure data.

Then process these failure data to get the reliability with the formula of medium-rank empirical value, lognormal distribution, Weibull distribution about two-parameter and probability distribution of maximum entropy (the formula would be shown in another paper on theory, the same as next), 
the vector quantity of empirical value on reliability $\mathrm{R} 0$ and the vector quantity of truth-value on reliability estimation R1, R2, R3 would be found out, and process with the formula of standard deviation.

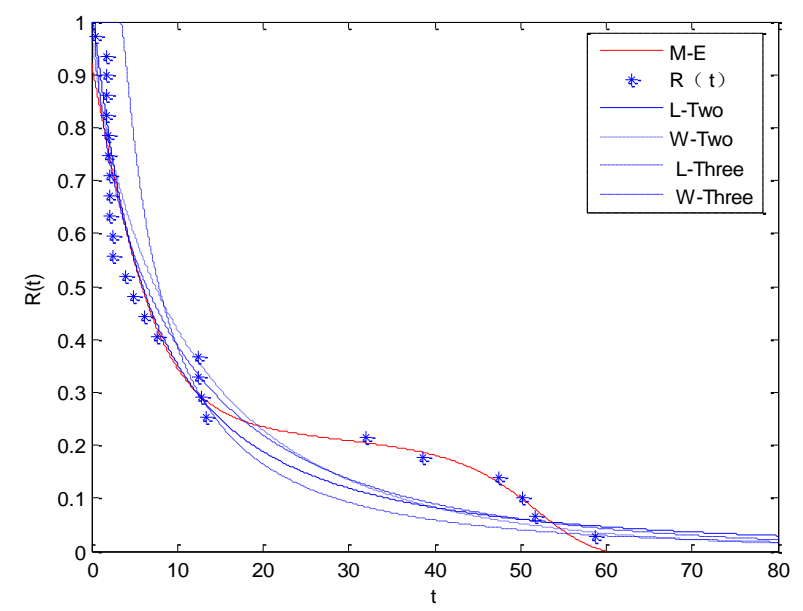

Figure 1. Reliability function graph of $\mathrm{T} 1$ failure data

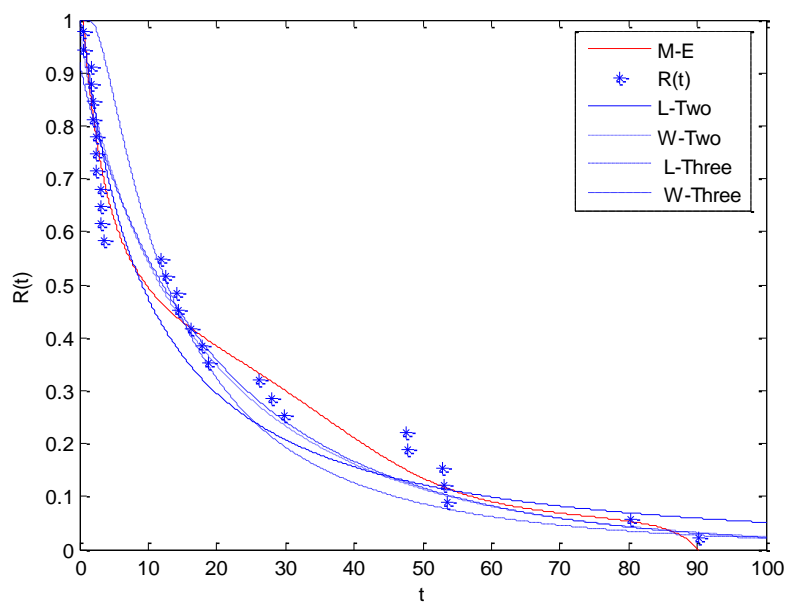

Figure 2. Reliability function graph of $\mathrm{T} 2$ failure data

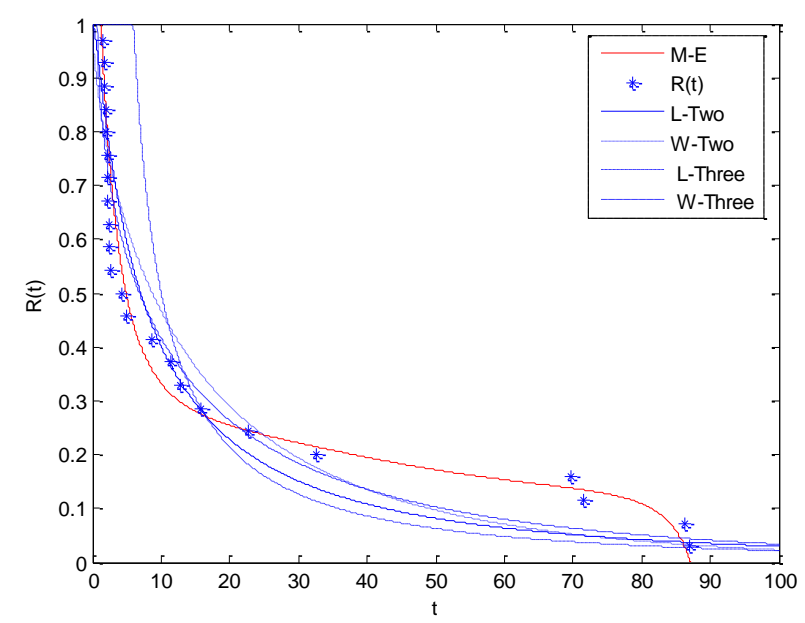

Figure 3. Reliability function graph of T3 failure data

Under the function of reliability $\mathrm{R}(t)=0.9$ or 0.5 , the fatigue life would be $\mathrm{P} 1$ or $\mathrm{P} 2$. Finally combine with the standard deviation to decide which one method has a better precision. 
Table 2 Comparative result of three reliability models for T1 failure data

\begin{tabular}{ccccccc}
\hline $\begin{array}{c}\text { Theoretical } \\
\text { method }\end{array}$ & $\begin{array}{c}\text { K-s } \\
\text { hypothesis } \\
\text { testing }\end{array}$ & $\begin{array}{c}\text { Critical } \\
\text { value }\end{array}$ & Yes or no & $\begin{array}{c}\text { Standard } \\
\text { deviation }\end{array}$ & P1 & P2 \\
\hline L-Two & 0.2024 & 0.2591 & yes & 0.0802 & 1.0295 & 5.9662 \\
W-Two & 0.2024 & 0.2591 & yes & 0.0899 & 0.6197 & 7.4173 \\
L-Three & 0.4615 & 0.2591 & no & nothing & nothing & nothing \\
W-Three & 0.1847 & 0.2591 & yes & 0.0773 & & \\
M-E & 0.1631 & 0.2591 & yes & 0.0737 & 0.2483 & 5.8670 \\
\hline
\end{tabular}

Table 3 Comparative results of three reliability models for T2 failure data

\begin{tabular}{ccccccc}
\hline $\begin{array}{c}\text { Theoretical } \\
\text { method }\end{array}$ & $\begin{array}{c}\text { K-s } \\
\text { hypothesis } \\
\text { testing }\end{array}$ & $\begin{array}{c}\text { Critical } \\
\text { value }\end{array}$ & Yes or no & $\begin{array}{c}\text { Standard } \\
\text { deviation }\end{array}$ & P1 & P2 \\
\hline L-Two & 0.1547 & 0.2417 & yes & 0.0757 & 1.3854 & 9.0933 \\
W-Two & 0.1696 & 0.2417 & yes & 0.0685 & 1.0675 & 11.7251 \\
L-Three & 0.3627 & 0.2417 & no & nothing & nothing & nothing \\
W-Three & 0.1795 & 0.2417 & yes & 0.0651 & & \\
M-E & 0.1140 & 0.2417 & yes & 0.0498 & 1.2390 & 9.7120 \\
\hline
\end{tabular}

Table 4 Comparative results of three reliability models for T3 failure data

\begin{tabular}{ccccccc}
\hline $\begin{array}{c}\text { Theoretical } \\
\text { method }\end{array}$ & $\begin{array}{c}\text { K-s } \\
\text { hypothesis } \\
\text { testing }\end{array}$ & $\begin{array}{c}\text { Critical } \\
\text { value }\end{array}$ & Yes or no & $\begin{array}{c}\text { Standard } \\
\text { deviation }\end{array}$ & P1 & P2 \\
\hline L-Two & 0.2400 & 0.2749 & yes & 0.0950 & 1.1562 & 7.0353 \\
W-Two & 0.2205 & 0.2749 & yes & 0.1008 & 0.5763 & 8.7075 \\
L-Three & 0.5652 & 0.2749 & no & nothing & nothing & nothing \\
W-Three & 0.1907 & 0.2749 & yes & 0.0831 & & \\
M-E & 0.1872 & 0.2749 & yes & 0.0702 & 1.6342 & 4.9040 \\
\hline
\end{tabular}

For the Table 2 4, these values of the k-s hypothesis testing for every theoretical methods expect the lognormal distribution of three-parameter are less than the critical value, that indicates four theoretical methods are suitable to analyze the reliability of failure data about rolling bearing. However, this viewpoint is right only based on the $\mathrm{k}-\mathrm{s}$ hypothesis testing, the location parameter $\tau$ is the minimum failure data, and should be less than zero. So the location parameter of Weibull distribution of three-parameter for $\mathrm{T} 2$ has no sense that shows its boundedness.

The D-value between the standard deviations of these theoretical methods is very tiny, that indicates these veracities. Whereas the value of P1 and P2 have a great difference between different methods, that cause the difference of fatigue life on rolling bearing.

In addition, the value of fatigue life ( $\mathrm{t}$ ) is one of the most important standard values under the function of reliability is $90 \%$ or $50 \%$. Based on the maximum entropy probability distribution because of the advantage of standard deviation, the relative error of fatigue life should be found out under the function of reliability is $90 \%$ or $50 \%$.

While the function of reliability is $90 \%$ :

The relative error of lognormal distribution of two-parameter as $f(\mathrm{~T} 1)$ :

$$
f(T 1)_{1}=\frac{1.0295-0.2483}{0.2483}=3.1458=314.62 \%
$$

While the function of reliability is $50 \%$ :

The relative error as $\mathrm{f}(\mathrm{T} 1)$ : 


$$
f(T 1)_{2}=\frac{5.9662-5.8670}{5.8670}=0.0169=1.69 \%
$$

The same as that, the relative error of these theoretical methods would be found out, and be shown in Table 5.

Table 5 Comparative results of the relative error for the different distributions of each group failure data

\begin{tabular}{ccccccc}
\hline $\begin{array}{c}\text { Relative } \\
\text { error }\end{array}$ & T1 L & T1 W & T2 L & T2 W & T3 L & T3 W \\
\hline$f_{1}$ & $314.62 \%$ & $149.58 \%$ & $11.81 \%$ & $13.84 \%$ & $29.25 \%$ & $64.74 \%$ \\
$f_{2}$ & $1.69 \%$ & $26.42 \%$ & $6.37 \%$ & $20.73 \%$ & $43.46 \%$ & $77.56 \%$ \\
\hline
\end{tabular}

As the Table 5 shows, these values of fatigue life have a numerous difference while the function of reliability is $90 \%$ or $50 \%$, and the maximum is $314.62 \%$ incredibly, even through these standard deviations are so close. The choice of the method has a significant effect to the veracity to predict the reliability about rolling bearing. Depend on the curve of function of reliability, a little change of $\mathrm{Y}$ would cause a enormous of $\mathrm{X}$. this study determines the degree of fitting of theoretical method with the standard deviation, so that induce the error of fatigue life.

\section{Summary}

According to the analysis of these theoretical methods, Weibull distribution has a better veracity than lognormal distribution generally and yet has an unstable performance. However, depending on the standard deviation and relative error, the maximum entropy probability distribution has an ideal veracity in the intercomparison of numerous theoretical methods, and haven't a perfect performance on all of the analysis all the same.

\section{Acknowledgements}

This project is supported by National Natural Science Foundation of China (Grant Nos. 51475144, 50375011 and 50675011).

\section{References}

[1] S.Norman. IEEE Trans. Vol.19 (1993) No.11, p.1095.

[2] A.J. Marcorin, and A.J. Abackerli: Quality and Reliability Engineering International.Vol.22 (2006) No.7, p.851.

[3] T.L. Zhang, and Y.K. Ren. Proceedings. (2002), p.44.

[4] D.X. Yang and L.B. Liu. Structural and Multidisciplinary Optimization. Vol.50 (2014) No.2, p.275.

[5] E.G. John and R.I. Grosvenor. Quality and Reliability Engineering International. Vol. 16 (2000) No.2, p.81.

[6] L. Podofillini and V.N. Dang. Reliability Engneering and System Safety. Vol.117 (2013), p.52.

[7] Y. Hao and G.W. Meng. Proceedings-2013 $4^{\text {th }}$ Intemational Conference on Intelligent System Design and Engineering Applications, ISDEA 2013. (2013), p.570.

[8] F. Li and G.W. Meng. Dongbei Daxue Xuebao/Journal of Northeastern University. Vol.34 (2013), p.288.

[9] D. Gonzalez-Gonzalez and C. Sifuentes. Engineering Application of Artificial Intelligence. Vol.32 (2014), p.151.

[10]X.T. Xia. Advanced Materials Research. Vol.488 (2012), p.1087. 